\title{
The Risk Factors for Asymptomatic Pyuria Among Patients With Type 2 Diabetes
}

\author{
Yoko Waragai $^{\mathrm{a}}$, Hidekatsu Yanaia, c, Tadayuki Kondo ${ }^{\mathrm{b}}$, Yu Takeuchi ${ }^{\mathrm{b}}$, \\ Tatsuro Hayakawa ${ }^{b}$, Akahito Sako ${ }^{a}$
}

\begin{abstract}
Background: Diabetic patients often develop urinary tract infections (UTI). Diabetic patients are also likely to develop mental illness, and psychotropic drugs have anticholinergic adverse effects such as urinary disturbance. We aimed to elucidate the risk factors of asymptomatic pyuria in patients with type 2 diabetes.
\end{abstract}

Methods: We picked up outpatients with type 2 diabetes who visited the Department of Internal Medicine for the first time, from December 2010 to November 2012 for the treatment of diabetes and completed the questionnaire to obtain the medical history. We obtained clinical information from medical records and questionnaires. Urine leukocytes $\geq 10$ /high-power field was defined as pyuria. Comparisons such as pyuria group vs. non-pyuria group and non-mental illness group vs. mood disorder group vs. schizophrenia group were performed.

Results: One hundred twenty-one patients were eligible. Sixty-nine were males, 52 were females, mean age was 63 years. There were 48 patients who had mental illness. The duration of diabetes was 5.1 years, hemoglobin A1c (HbA1c) was $8.5 \%$. Prevalence of pyuria was $15.7 \%$. Mean age in non-pyuria group was 62.8 years old, and 65.1 years old in pyuria group. Proportion of female (84\%) in pyuria group was significantly higher than non-pyuria group. Patients with pyuria significantly more often had mental illness and psychotropic drugs prescription than patients without pyuria. Duration of diabetes in non-pyuria group was 5.3 years and that in pyuria group was 4.0 years. Coefficient of variations of R-R intervals (CVR-R) in electrocardiogram (ECG), an index of autonomic nervous dysfunction, was $3.2 \%$ in non-pyuria group and $1.8 \%$ in pyuria group. Pyuria group had significantly higher prevalence of autonomic nervous dysfunction. Coexistence of mental illness and lower CVR-R were associated with risk of pyuria in female diabetic patients.

Manuscript submitted December 6, 2019, accepted December 13, 2019

${ }^{a}$ Department of Internal Medicine, Kohnodai Hospital, National Center for Global Health and Medicine, Chiba, Japan

bepartment of Psychiatry, National Center for Global Health and Medicine Kohnodai Hospital, Chiba, Japan

${ }^{\mathrm{c} C o r r e s p o n d i n g ~ A u t h o r: ~ H i d e k a t s u ~ Y a n a i, ~ D e p a r t m e n t ~ o f ~ I n t e r n a l ~ M e d i c i n e, ~}$ National Center for Global Health and Medicine Kohnodai Hospital, 1-7-1 Kohnodai, Ichikawa, Chiba 272-8516, Japan.

Email: dyanai@hospk.ncgm.go.jp

doi: https://doi.org/10.14740/jem629
Conclusions: In addition to female sex, autonomic neuropathy and mental illness were significant risk factors for pyuria in patients with type 2 diabetes. To elucidate this association, further studies, preferably with larger numbers of subjects, will be needed.

Keywords: Autonomic nervous dysfunction; Diabetes; Mental illness; Pyuria

\section{Introduction}

Urinary tract infections (UTI) such as pyelonephritis and cystitis are common in diabetic patients. Various impairments in the immune system, poor metabolic control, and incomplete bladder emptying due to autonomic neuropathy may contribute to the enhanced risk of UTI in diabetic patients [1].

Sodium glucose cotransporter-2 (SGLT-2) inhibitors, which promote excretion of glucose into urine, have been used since 2014 in Japan. We will face the increase of UTI in patients with diabetes.

Ten to thirty percent of patients with diabetes have asymptomatic pyuria $[2,3]$. Female, aging, longer duration of diabetes and presence of diabetic complications are considered as risk factors in previous studies [2, 3]. However, there have been only a few studies.

Diabetic patients often develop mental illness. Psychotropic drugs have anticholinergic adverse effects such as urinary disturbance [4]. However, there are no studies that investigated the association between mental illness and pyuria.

We aimed to elucidate the risk factors for asymptomatic pyuria in patients with type 2 diabetes, and identify the patients who should be paid attention for the occurrence of UTI.

\section{Patients and Methods}

This study is a retrospective observational study. We picked up outpatients with type 2 diabetes who visited the Department of Internal Medicine for the first time, from December 2010 to November 2012 for the treatment of diabetes and completed the questionnaire to obtain the medical history. We excluded patients who did not have urinary sediment data at the first visit, and patients who had used antibiotics within 1 month and patients who already had cystitis or pyelonephritis at the 


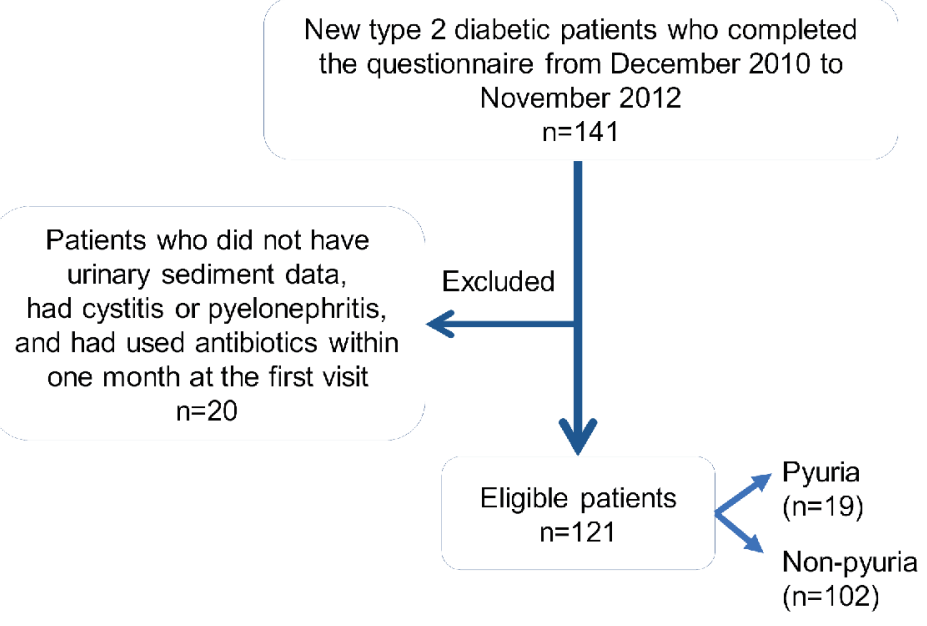

Figure 1. The recruitment of studied subjects.

first visit.

We obtained some clinical information from medical records and questionnaires; patient's backgrounds (age, gender, height, weight, body mass index (BMI), medication, coexisting illness, mental illness); diabetic complications (retinopathy, nephropathy, neuropathy assessed by using coefficient of variations of R-R intervals (CVR-R) in electrocardiogram (ECG)); blood and urine tests. We used CVR-R as the marker for autonomic nervous dysfunction. The variations of the time interval between heartbeats are physiological. The decrease of CVR-R suggests the presence of autonomic nervous disturbance. According to the previous study [5], we adopted more than $2 \%$ as the normal range of CVR-R.

Urine leukocytes $\geq 10$ /high-power field was defined as pyuria [2]. Comparisons such as pyuria group vs. non-pyuria group and non-mental illness group vs. mood disorder group vs. schizophrenia group were performed. Chi-square test, $t$ test, Fisher exact probability test, analysis of variance, GamesHowell multiple comparison were used. $\mathrm{P}<0.05$ was considered to be statistically significant. Statistical software IBM SPSS Statistics 22 was used.

This study was approved by the Institutional Ethics Committee in National Center for Global Health and Medicine (NCGM-G-001527), and was also performed in accordance with the Declaration of Helsinki.

\section{Results}

The recruitment of studied subjects was shown in Figure 1. In 141 patients who visited the Department of Internal Medicine for the first time from December 2010 to November 2012 for the management of diabetes, 20 patients were excluded, 121 were eligible. Characteristics of studied subjects were shown in Table 1. Sixty-nine were males, 52 were females, mean age was 63 years old. There were 48 patients who had mental illness. The duration of diabetes was 5.1 years, hemoglobin A1c (HbA1c) was $8.5 \%$. Prevalence of pyuria was $15.7 \%$.
Comparison of clinical characteristics between non-pyuria group and pyuria group was shown in Table 2. Mean age in non-pyuria group was 62.8 years old, and 65.1 years old in pyuria group, and there was no significant difference. Proportion of female was $84 \%$ in pyuria group. This was significantly higher than non-pyuria group. Patients with pyuria significantly more often had mental illness and psychotropic drugs prescription than patients without pyuria.

Comparison of diabetic complications between non-pyuria group and pyuria group was shown in Table 3. Duration of diabetes in non-pyuria group was 5.3 years and that in pyuria group was 4.0 years. CVR-R in pyuria group (1.8\%) was sig-

Table 1. Characteristics of Studied Subjects

\begin{tabular}{ll}
\hline Gender (male/female) & $69 / 52$ \\
\hline Age (year) & $63.2 \pm 13.4$ \\
Weight $(\mathrm{kg})$ & $67.0 \pm 16.2$ \\
BMI $\left(\mathrm{kg} / \mathrm{m}^{2}\right)$ & $26.0 \pm 5.7$ \\
Mental illness (n) & 48 \\
\hline Duration of diabetes (year) & $5.1 \pm 7.5$ \\
HbA1c $(\%)$ & $8.5 \pm 2.1$ \\
Plasma glucose (mg/dL) & $197 \pm 95$ \\
Urine leukocytes (/HPF) & \\
\hline$<1$ & 66 \\
1 - 4 & 25 \\
5 - 9 & 11 \\
$10-29$ & 11 \\
30 - 49 & 4 \\
50 - 99 & 3 \\
$\geq 100$ & 1 \\
\hline Total & 121 \\
\hline
\end{tabular}

BMI: body mass index; HbA1c: hemoglobin A1c; HPF: high-power field. 
Table 2. Comparison of Clinical Characteristics Between Non-Pyuria Group and Pyuria Group

\begin{tabular}{llll} 
& Non-pyuria $(\mathbf{n}=102)$ & Pyuria $(\mathbf{n}=\mathbf{1 9})$ & P value \\
\hline Age (year) & $62.8 \pm 12.7$ & $65.1 \pm 17.2$ & 0.60 \\
$\geq 65$ years old $(\%)$ & 51.0 & 63.2 & 0.33 \\
Female $(\%)$ & 35.3 & 84.2 & $<0.01$ \\
Weight $(\mathrm{kg})$ & $67.3 \pm 16.4$ & $65.3 \pm 15.5$ & 0.62 \\
Waist circumference $(\mathrm{cm})(\mathrm{n}=99)$ & 92.1 & 96.1 & 0.32 \\
BMI $\left(\mathrm{kg} / \mathrm{m}^{2}\right)$ & $25.6 \pm 5.6$ & $27.7 \pm 6.2$ & 0.16 \\
Mental illness $(\%)$ & 35.3 & 63.2 & 0.02 \\
Taking psychotropic drugs $(\%)$ & 33.3 & 57.9 & 0.04 \\
Duration of mental illness (year) & $17.7 \pm 14.3$ & $16.9 \pm 17.0$ & 0.85 \\
\hline
\end{tabular}

BMI: body mass index.

Table 3. Comparison of Diabetic Complications Between Non-Pyuria Group and Pyuria Group

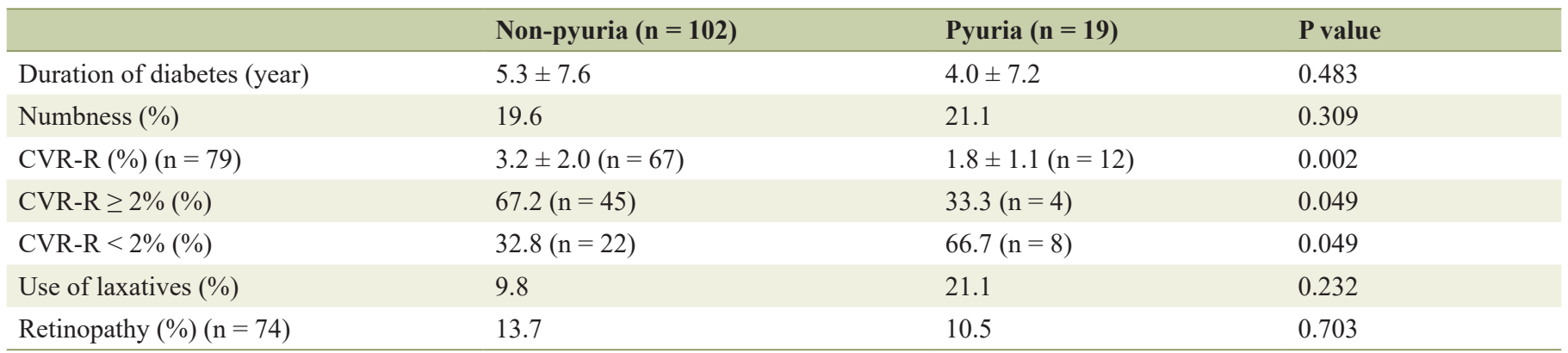

CVR-R: coefficient of variations of $R-R$ intervals.

nificantly lower than in non-pyuria group (3.2\%). We adopted the cut-off value of lower than $2 \%$ in CVR-R to determine the autonomic nervous dysfunction [5]. Patients with pyuria significantly more often had autonomic nervous dysfunction than patients without pyuria. There were no significant differences in the symptom of numbness, use of laxatives and retinopathy between two groups.

Comparison of laboratory data between non-pyuria group and pyuria group was shown in Table 4. Glucosuria, plasma glucose, HbA1c were similar between two groups. Comparison of data between non-pyuria group and pyuria group in female patients was shown in Table 5. We analyzed data in female alone because female sex was a possible cofounding factor. Coexistence of mental illness and low CVR-R were significantly associated with the development of pyuria in female diabetic patients.

Coexisting mental illness in studied subjects were shown in Figure 2. Proportion of patients with mental illness was $40 \%$. Nineteen percent was schizophrenia and $17 \%$ was mood disorder. Differences in clinical characteristics among patients with non-mental illness, mood disorder and schizophrenia were shown in Table 6. Prevalence of pyuria was $21 \%$ in schizophrenia group; however, the difference was not significant. The proportions of female were not different among three groups. Schizophrenia patients were younger and had higher BMI.

Differences in clinical characteristics among patients with

Table 4. Comparison of Laboratory Data Between Non-Pyuria Group and Pyuria Group

\begin{tabular}{llll} 
& Non-pyuria $(\mathbf{n}=\mathbf{1 0 2})$ & Pyuria $(\mathbf{n}=\mathbf{1 9})$ & P value \\
\hline eGFR (mL/min) & $82.1 \pm 21.8$ & $72.6 \pm 25.7$ & 0.10 \\
Cre (mg/dL) & $0.72 \pm 0.17$ & $0.74 \pm 0.3$ & 0.67 \\
BUN (mg/dL) & $14.6 \pm 5.3$ & $14.5 \pm 5.0$ & 0.92 \\
Proteinuria (\%) & 16.7 & 31.6 & 0.20 \\
Glucosuria (\%) & 42.2 & 42.1 & 1.00 \\
Plasma glucose (mg/dL) & $199 \pm 100$ & $190 \pm 63$ & 0.70 \\
HbA1c (\%) (n=120) & $8.5 \pm 2.2$ & $8.4 \pm 1.5$ & 0.80 \\
\hline
\end{tabular}

Cre: creatinine; eGFR: estimated glomerular filtration rate; BUN: blood urea nitrogen; HbA1c: hemoglobin A1c. 
Table 5. Comparison of Data Between Non-Pyuria Group and Pyuria Group in Female Patients

\begin{tabular}{llll}
\hline & $\begin{array}{l}\text { Non-pyuria } \\
(\mathbf{n = 3 6 )}\end{array}$ & $\begin{array}{l}\text { Pyuria } \\
(\mathbf{n = 1 6 )}\end{array}$ & P value \\
\hline Mental illness (\%) & 36.1 & 75.0 & 0.01 \\
CVR-R $<2 \%(\%)$ & 18.2 & 72.7 & $<0.01$ \\
CVR-R (\%) & 3.5 & 1.5 & $<0.01$ \\
\hline
\end{tabular}

CVR-R: coefficient of variations of R-R intervals.

non-mental illness, mood disorder and schizophrenia were shown in Table 7. Schizophrenia patients more often used laxatives, which suggested the existence of diabetic neuropathy, however, CVR-R were not different. There were no significant differences in plasma glucose and HbA1c among three groups.

\section{Discussion}

The number of studies on the risk factors of asymptomatic pyuria in patients with type 2 diabetes was very limited. Comparison of results between previous studies and present study was shown in Table $8[2,3]$. Nakano et al studied only female patients, and found that diabetic women more often had asymptomatic pyuria than non-diabetic women $(27.9 \%$ vs. $15.9 \%, \mathrm{P}<0.001)$ [2]. They also found that the prevalence of asymptomatic pyuria was significantly increased in patients with duration of diabetes exceeding 15 years. In their study, diabetic women with asymptomatic pyuria more often had diabetic retinopathy, neuropathy, nephropathy, cerebrovascular disease, ischemic heart disease, and hyperlipidemia than those without asymptomatic pyuria, and the incidence of asympto- matic pyuria increases significantly as diabetic microangiopathy becomes severer [2]. However, we did not observe a significant association of asymptomatic pyuria with the duration of diabetes, incidence and severity of diabetic microangiopathy except for CVR-R. The duration of diabetes in our patients was shorter than that in Nakano's study, which can explain the different results between ours and their study. Nakano et al [2] did not find differences in $\mathrm{HbA} 1 \mathrm{c}$ between diabetic patients without asymptomatic pyuria and those with asymptomatic pyuria, which agreed with our result.

Ozdem et al investigated the prevalence of asymptomatic pyuria in 227 diabetic patients, and found that diabetic women and men had significantly higher asymptomatic pyuria prevalence than non-diabetic women $(21.4 \%$ vs. $8.7 \%, \mathrm{P}<0.05)$ and men $(12.2 \%$ vs. $3.4 \%, \mathrm{P}<0.05)$, suggesting that diabetes was the risk factor for asymptomatic pyuria [3]. They also reported that the prevalence of pyuria was markedly higher in diabetic women as compared with diabetic men $(\mathrm{P}=0.08)$, which agreed with our result. In their study, diabetes duration and HbA1c levels were similar in diabetic patients with and without asymptomatic pyuria [3], which also agreed with our data.

Our study is the first to report a significant association between mental illness and pyuria in patients with type 2 diabetes. Diabetic patients with pyuria more often had mental illness than those without pyuria $(63.2 \%$ vs. $35.3 \%, \mathrm{P}=0.02)$, and patients with pyuria more often had taken psychotropic drugs than those without pyuria $(57.9 \%$ vs. $33.3 \%, \mathrm{P}=0.04)$. Difficulty in the maintenance of hygienic conditions and psychotropic drugs which induce urinary disturbance may be associated with more frequent development of pyuria in patients with mental illness [4].

Present study also is the first to show that lower CVR-R, which reflects diabetic autonomic neuropathy, was a significant risk factor of pyuria. In the recent review article [6], the

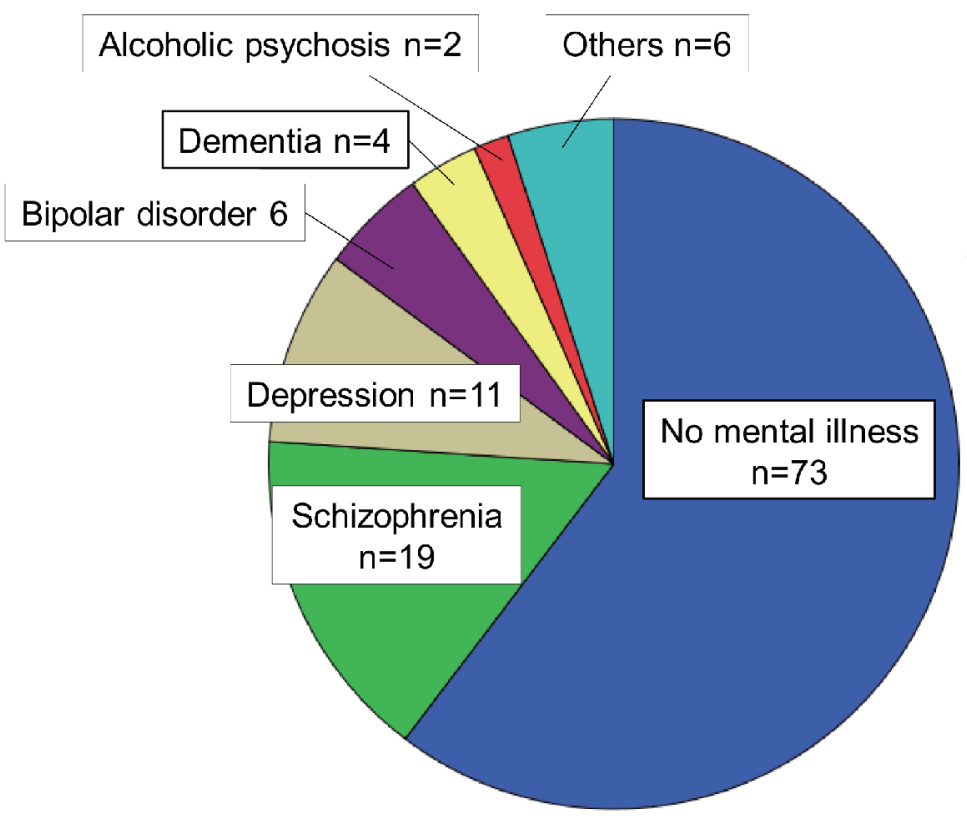

Figure 2. Coexisting mental illness in studied subjects. 
Table 6. Differences in Clinical Characteristics Among Patients With Non-Mental Illness, Mood Disorder and Schizophrenia

\begin{tabular}{lllll}
\hline & Non-mental illness $(\mathbf{n}=\mathbf{7 3})$ & Mood disorder $(\mathbf{n}=\mathbf{1 7})$ & Schizophrenia $(\mathbf{n}=\mathbf{1 9})$ & P value \\
\hline Pyuria $(\%)$ & 9.6 & 11.8 & 21.1 & 0.36 \\
Age (year) & $66.1 \pm 10.9$ & $58.7 \pm 16.5$ & $56.1 \pm 15.1$ & $<0.01$ \\
$\geq 65$ years old $(\%)$ & 56.2 & 52.9 & 42.1 & 0.55 \\
Female $(\%)$ & 37.0 & 47.1 & 47.4 & 0.59 \\
BMI $\left(\mathrm{kg} / \mathrm{m}^{2}\right)$ & $24.5 \pm 4.2$ & $28.4 \pm 7.8$ & $30.3 \pm 6.7$ & $<0.01$ \\
\hline
\end{tabular}

BMl: body mass index.

Table 7. Differences in Clinical Characteristics Among Patients With Non-Mental Illness, Mood Disorder and Schizophrenia

\begin{tabular}{lllll}
\hline & Non-mental illness $(\mathbf{n}=\mathbf{7 3})$ & Mood disorder $(\mathbf{n}=\mathbf{1 7})$ & Schizophrenia $(\mathbf{n}=\mathbf{1 9})$ & P value \\
\hline CVR-R (\%) $(\mathrm{n}=60)$ & $3.1 \pm 2.1$ & $2.8 \pm 1.9$ & $3.6 \pm 2.2$ & 0.71 \\
Use of laxatives (\%) & 4.1 & 11.8 & 26.3 & 0.01 \\
Retinopathy (\%) $(\mathrm{n}=74)$ & 11 & 23.5 & 10.5 & 0.72 \\
Glucosuria (\%) & 41.7 & 29.4 & 57.9 & 0.22 \\
Plasma glucose (mg/dL) & $198 \pm 92$ & $203 \pm 132$ & $207 \pm 79$ & 0.93 \\
HbA1c (\%) & $8.6 \pm 2.1$ & $8.8 \pm 2.5$ & $8.5 \pm 2.0$ & 0.92 \\
\hline
\end{tabular}

CVR-R: coefficient of variations of R-R intervals; HbA1c: hemoglobin A1c.

potential relationships between the autonomic nervous system and UTI are considered throughout. While these relationships have not been experimentally explored, the known interactions of UTI characteristics including symptoms, complicating factors, and inflammation with autonomic nervous system function suggest a significant association between autonomic nervous system and UTI. However, the definitive conclusion has not yet been drawn even regarding the relationship between UTI and autonomic nervous system. There was no study on the association between asymptomatic pyuria and autonomic dysfunction. A significant association between lower CVR-R and asymptomatic pyuria observed in present study should be followed by using a great number of patients in the future.

We have to mention the limitations of present study. Small sample size and missing data involving CVR-R were considered to be the limitations. In addition, analyzing the asymptomatic pyuria which surrogated the endpoint of UTI was considered to be the limitation. And we could not discriminate between pollakiuria due to diabetes and cystitis.

\section{Conclusions}

We studied the risk factors for asymptomatic pyuria in patients with type 2 diabetes. In addition to female sex, autonomic neuropathy and mental illness were significant risk factors for pyuria. To elucidate this association, further studies, preferably with larger numbers of subjects, will be needed.

Table 8. Comparison of Results Between Previous Studies And Present Study

\begin{tabular}{llll}
\hline Studies & Nakano et al, [2] & Ozdem et al, [3] & Present study \\
\hline Publication (year) & 2001 & 2006 & 2019 \\
Mean age (years) & 64.8 & 60.1 & 63.2 \\
HbAlc (\%) & 8.6 & 9.1 & 8.5 \\
Duration of diabetes (years) & 10.0 & 7.9 & 5.1 \\
Prevalence of pyuria (\%) & & 3.4 & - \\
$\quad$ Non-diabetic males & - & 8.7 & - \\
$\quad$ Non-diabetic females & 15.8 & 12.2 & 4.3 \\
\multicolumn{1}{c}{ Diabetic males } & - & 21.4 & 30.8 \\
$\quad$ Diabetic females & 27.9 & Diabetes, female & Mental illness, female, \\
Risk factors & Older age, diabetes, retinopathy, neuropathy, nephropathy, & lower CVR-R \\
\hline
\end{tabular}

CVR-R: coefficient of variations of R-R intervals; HbA1c: hemoglobin A1c. 


\section{Acknowledgments}

We thank the staffs (Yukie Kawamura, Keiko Nakamura, Harue Aoki and Ayano Sakakibara) of the Division of Research Support, National Center for Global Health and Medicine Kohnodai Hospital.

\section{Financial Disclosure}

Authors have no financial disclosure to report.

\section{Conflict of Interest}

The authors declare that they have no conflict of interest concerning this article.

\section{Informed Consent}

Not applicable.

\section{Author Contributions}

AS, TK, YT and YW designed the research. TK, YT and YW collected data. AS, TH and HY analyzed data, and HY wrote the paper. All authors read and approved the final paper.

\section{References}

1. Nitzan O, Elias M, Chazan B, Saliba W. Urinary tract infections in patients with type 2 diabetes mellitus: review of prevalence, diagnosis, and management. Diabetes Metab Syndr Obes. 2015;8:129-136.

2. Nakano H, Oba K, Saito Y, Ouchi M, Yamashita N, Okamura K, Takai E, et al. Asymptomatic pyuria in diabetic women. J Nippon Med Sch. 2001;68(5):405-410.

3. Ozdem S, Bayraktar T, Oktay C, Sari R, Gultekin M. The prevalence of asymptomatic pyuria in diabetic patients: comparison of the Sysmex UF-100 automated urinalysis analyzer with Fuchs-Rosenthal hemacytometer. Clin Biochem. 2006;39(9):873-878.

4. Faure Walker N, Brinchmann K, Batura D. Linking the evidence between urinary retention and antipsychotic or antidepressant drugs: A systematic review. Neurourol Urodyn. 2016;35(8):866-874.

5. Kageyama S, Taniguchi I, Tanaka S, Tajima N, Saito N, Ikeda Y, Abe M. A critical level of diabetic autonomic neuropathy. Tohoku J Exp Med. 1983;141(Suppl):479-483.

6. Hibbing ME, Conover MS, Hultgren SJ. The unexplored relationship between urinary tract infections and the autonomic nervous system. Auton Neurosci. 2016;200:29-34. 